\title{
TRACE: A Differentiable Approach to Line-Level Stroke Recovery for Offline Handwritten Text
}

Taylor Neil Archibald

Brigham Young University

Follow this and additional works at: https://scholarsarchive.byu.edu/etd

Part of the Computer Sciences Commons

\section{BYU ScholarsArchive Citation}

Archibald, Taylor Neil, "TRACE: A Differentiable Approach to Line-Level Stroke Recovery for Offline Handwritten Text" (2020). Theses and Dissertations. 9299.

https://scholarsarchive. byu.edu/etd/9299

This Thesis is brought to you for free and open access by BYU ScholarsArchive. It has been accepted for inclusion in Theses and Dissertations by an authorized administrator of BYU ScholarsArchive. For more information, please contact ellen_amatangelo@byu.edu. 
TRACE: A Differentiable Approach to Line-Level Stroke Recovery for Offline Handwritten Text

Taylor Archibald

A thesis submitted to the faculty of Brigham Young University in partial fulfillment of the requirements for the degree of

Master of Science

Tony Martinez, Chair

Bryan Morse

Parris Egbert

Department of Computer Science

Brigham Young University

Copyright (C) 2020 Taylor Archibald

All Rights Reserved 


\title{
ABSTRACT \\ TRACE: A Differentiable Approach to Line-Level Stroke Recovery for Offline Handwritten Text \\ Taylor Archibald \\ Department of Computer Science, BYU \\ Master of Science
}

\begin{abstract}
Stroke order and velocity are helpful features in the fields of signature verification, handwriting recognition, and handwriting synthesis. Recovering these features from offline handwritten text is a challenging and well-studied problem. We propose a new model called TRACE (Trajectory Recovery by an Adaptively-trained Convolutional Encoder). TRACE is a differentiable approach using a convolutional recurrent neural network (CRNN) to infer temporal stroke information from long lines of offline handwritten text with many characters. TRACE is perhaps the first system to be trained end-to-end on entire lines of text of arbitrary width and does not require the use of dynamic exemplars. Moreover, the system does not require images to undergo any pre-processing, nor do the predictions require any post-processing. Consequently, the recovered trajectory is differentiable and can be used as a loss function for other tasks, including synthesizing offline handwritten text.
\end{abstract}

We demonstrate that temporal stroke information recovered by TRACE from offline data can be used for handwriting synthesis and establish the first benchmarks for a stroke trajectory recovery system trained on the IAM online database.

Keywords: handwriting, stroke trajectory recovery, deep learning 


\section{Table of Contents}

List of Figures $\quad$ V

List of Tables $\quad$ vi

1 Introduction $\quad 1$

2 Related Work $\quad 3$

2.1 Historical approaches to stroke recovery . . . . . . . . . . . . . 3

2.2 Deep learning approaches to stroke recovery . . . . . . . . . . . . . . 4

3 Method $\quad 6$

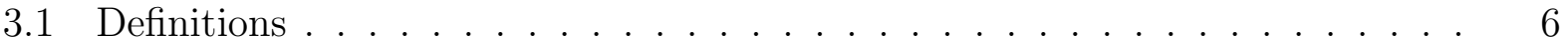

3.2 Loss . . . . . . . . . . . . . . . . . . . . . . . 7

3.3 Adaptive Ground Truth . . . . . . . . . . . . . . . . . . . 9

3.4 Encoder-Decoder Network . . . . . . . . . . . . . . . . . . . 12

4 Implementation $\quad 16$

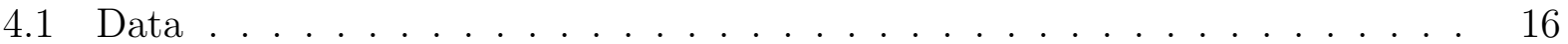

5 Experiments $\quad 18$

5.1 Online evaluation . . . . . . . . . . . . . . . . . . . . 18

5.2 Offline evaluation . . . . . . . . . . . . . . . . 20

5.3 Synthesis evaluation . . . . . . . . . . . . . . . . . 21

5.4 Synthetic Evaluation . . . . . . . . . . . . . . . . . . . . . 24 
5.5 Adaptive GT Ablation Study . . . . . . . . . . . . . . . . . . . . . . 24

6 Conclusion $\quad 27$

$\begin{array}{lr}\text { References } & 28\end{array}$ 


\section{List of Figures}

1.1 TRACE recovery and synthesis . . . . . . . . . . . . . . . 2

3.1 The network architecture for TRACE . . . . . . . . . . . . . . 13

3.2 Our CNN architecture . . . . . . . . . . . . . . . . . 14

5.1 Random sample of IAM-offline stroke reconstructions . . . . . . . . . . . 19

5.2 TRACE achieves robust performance despite the presence of an anomalous marking. . . . . . . . . . . . . . . . . . . . . . . 21

5.3 Synthetic online handwriting samples inspired from offline styles . . . . . . . 23

5.4 Comparison of synthesized text based on different training data . . . . . . 23

5.5 The number of GT adaptive improvements per training instance converges. . $\quad 25$

5.6 Adapting GTs on the training set improves NN loss on test set. . . . . . . 26 


\section{List of Tables}

5.1 Average DTW distance . . . . . . . . . . . . . . . . 19

5.2 Online $\mathrm{NN}$ distance . . . . . . . . . . . . . . . . . . . . . . . . . . . . . 19

5.3 Offline $\mathrm{NN}$ distance . . . . . . . . . . . . . . . . . . . . . . 20 


\section{Chapter 1}

\section{Introduction}

Handwriting is prevalent in both the physical and digital world. When handwriting is captured by a digital device, such as a pen-based computer screen, it is referred to as online handwriting data. At a minimum, these data include the location of the pen tip or stylus when touching the screen through time [19]. On the other hand, offline handwriting data refers to digital images of handwriting inscribed on some physical medium. While both are representations of the same idea, there are important distinctions in how they can be used.

While online data can be readily rendered as an image, the reverse process is much more difficult, as offline data lack a temporal component and often contain artifacts inherent to the writing medium or digitization process. Consequently, online handwriting data can make many tasks easier or more accurate [18], including handwriting recognition, signature verification, writer identification, and handwriting synthesis (see Figure 1.1). While capturing handwriting online is becoming increasingly common, processing and leveraging offline handwriting data remains an important challenge.

While distinct tools and methods have been adapted to both domains, if data could be robustly translated from one domain to the other, systems designed for either domain could natively process data from the other, while simultaneously benefitting from having data from the other domain available to augment existing training data. Recovering online information from offline images is half of this challenge and has been a point of interest for research for several decades. In this case, if stroke order could be reliably recovered from offline handwriting data, our ability to perform most handwriting tasks would improve [20]. 

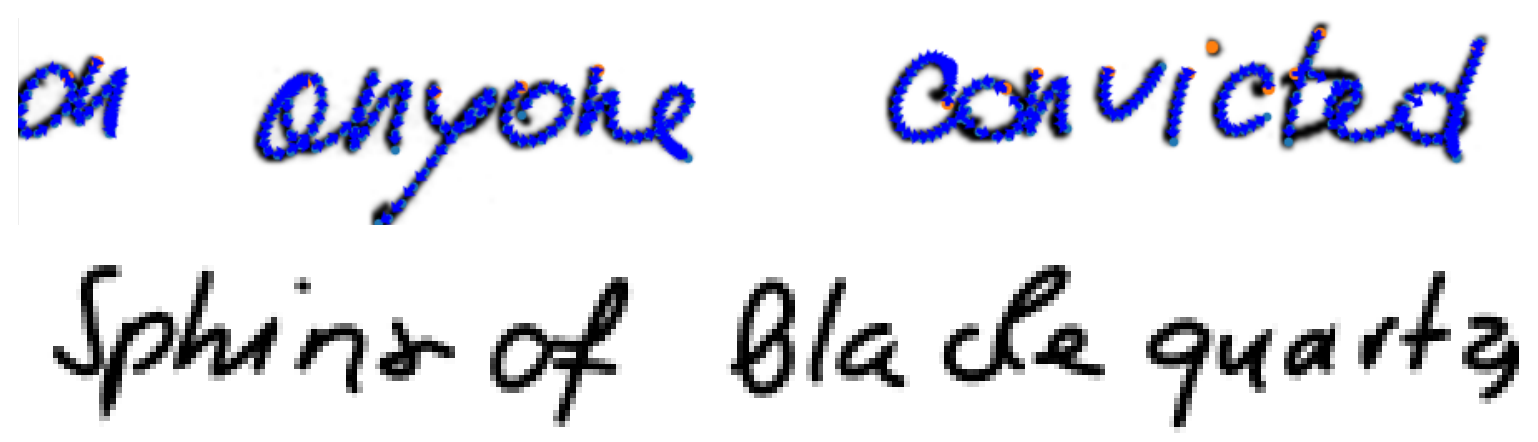

Figure 1.1: TRACE recovery and synthesis. (1) is a visualization of strokes recovered by TRACE from an offline handwriting image. Blue arrows indicate the predicted direction and orange points indicate the beginning of a new stroke. (2) is an example of a synthetically generated image that mimics the style of (1), and demonstrates how strokes recovered from offline data can be used for other tasks.

This is evidenced in part by the fact that many modern online handwriting recognition systems make explicit use of either the stroke order or the time component of the data when it is available $[4,11,21]$.

We propose a novel, differentiable technique for stroke recovery called TRACE (Trajectory Recovery by an Adaptively-trained Convolutional Encoder). A deep convolutional neural network $(\mathrm{CNN})$ is used to extract features from an image of a line of offline text of arbitrary width. These features represent a sequential, left to right encoding of the image, which is then passed into a recurrent neural network (RNN). The RNN outputs a series of predicted stroke points, the number of which is proportional to the width of the original image. These predictions are then compared against the ground truth (GT) stroke points to calculate a loss, from which the network is updated.

TRACE offers many advantages relative to prior approaches to this problem. First, TRACE is fully differentiable. The advantage of this is that it can be used as part of a loss function for other tasks, including synthesizing offline handwritten images. Moreover, after handwriting has been appropriately resized and segmented into lines, our method requires no further preprocessing or skeletonization, processing that could lose information that might be helpful for inferring stroke order, direction, or velocity. Furthermore, our approach is well-adapted to work on arbitrarily long lines of text. 


\section{Chapter 2}

\section{Related Work}

Stroke recovery has been researched for over 50 years. Various methods, including explicit methods, hidden markov models (HMMs), and deep neural networks have all been used with mixed success.

\subsection{Historical approaches to stroke recovery}

Traditional, explicit methods broadly split the problem into two phases: local examination, where strokes are analyzed for startpoints, endpoints, loops, ambiguous zones, and other complications, and global reconstruction, where the strokes are reconstructed based on features and observations derived from local examination [17].

Some of the earliest works in handwriting trajectory recovery first reduced an image to a skeleton, a one-pixel-width rendering of the text. While this skeleton should correspond perfectly to the original stroke, traditional methods of thinning are sensitive to noise and are prone to create artificial splits or misrepresent blobs (hidden loops) [17]. While there exist techniques for resolving these issues, the general problem with skeletonization is that information that might be used to infer velocity or trajectory is being discarded.

After preprocessing, ambiguous zones, where strokes intersect or where start/end points are ambiguous, are detected and analyzed, often from a set of heuristic rules [17]. While these methods established respectable accuracy, the process of deriving many of the heuristics used is time-consuming and variously character-set specific. 
Another method of stroke recovery involves a Hidden Markov Model (HMM) $[2,16]$. An HMM is a probabilistic model that assumes the system being modeled has some unobservable, "hidden" state, with probabilistic transitions from one state to another, with these states having some probabilistic effect on the observed outcomes. In the case of stroke recovery, the sequence of pen positions needed to produce the image are the hidden states. The image is first skeletonized, before feeding the data into the HMM, which attempts to recover the most likely trajectory given the model and provided skeleton. The HMM provides a nice generative framework with an explicit structure and defined relationships between states that can be used with limited training data. However, the optimal number of hidden states is not known a priori. Moreover, the rigid definition of state transitions can limit the model's ability to generalize on complex or long-term dependencies.

\subsection{Deep learning approaches to stroke recovery}

The first published attempt to apply deep learning to end-to-end stroke recovery from an image is perhaps [13]. Using an encoder-decoder style network, Bhunia et al. employed a CNN followed by a particular type of recurrent neural network known as a "Long-Short Term Memory" (LSTM) [10]. Benefits of using an LSTM include that it can have an arbitrary number of states and can learn long-term dependencies. They demonstrate its effectiveness on single stroke characters with square images.

In [25], Zhao et al. use a CNN and dynamic energy prediction network for Chinese single-character recognition. In [23], Sumi et al. use a Cross Variational Autoencoder to translate from offline to online characters and vice versa. The process involves learning a shared latent space between online and offline representations of characters, by iteratively passing online data to one encoder and offline data to another encoder. Representations encoded in this latent space are then decoded into both an online and offline data representation, where the difference between the reconstruction and ground truth is used to tune the network. 
However, these efforts have all focused on trajectory recovery for single characters and may not be easily adapted to handle variable width images consisting of many characters. 


\section{Chapter 3}

\section{Method}

Our goal is to leverage deep learning's image pattern recognition ability to recover strokes from arbitrarily wide lines of text of the kind often used in the field of handwriting recognition.

\subsection{Definitions}

We define a stroke as the function interpolated from a sequence of points that begins with a "pen down" action and terminates with a "pen up" action. We refer to a line of text as one or more strokes, where additional characters are expanded along the horizontal axis.

We can represent a sequence of strokes as a parametric function $r(d)=(x(d), y(d))$, where $d$ is the cumulative stroke distance (i.e., the total distance the pen has travelled), and $x$ and $y$ are coordinates in the Cartesian plane. Our goal is to find some approximate function for $r(d), r^{\prime}(d)$, given some input image $I$. One way of approximating this function is to find a series of points $P$ that are proximate to the set of points $T$, sampled from $r(d)$ at regular intervals. That is, for some number of stroke points $n$, we generate a set of target stroke points

$$
T=\left\{r\left(d_{i}\right)\right\} \text { for } d_{i} \in\left\{0,1 \frac{d_{\max }}{n-1}, 2 \frac{d_{\max }}{n-1}, \ldots, d_{\max }\right\}
$$

where $d_{0}=0$ and $d_{i}$ is sampled at regular intervals of $\frac{d_{\max }}{n-1}$. 
We can then measure the distance between these target points $T$ and our predictions $P$ according to some mapping, to evaluate our approximation of $r$.

\subsection{Loss}

Our loss function should favor stroke point combinations that could have generated the original image. This means that all predicted stroke points should lie somewhere on the original strokes, and they should collectively cover the entirety of the original strokes. We also desire for the model to accurately predict both the order the strokes were originally written as well as the direction of each stroke.

In [13], the authors employed an $L_{1}$ loss, or the Manhattan distance from the predicted stroke points to the ground-truth points, where each predicted point is mapped to the GT point with the same index in the sequence. However, this approach has a number of considerable drawbacks. For instance, when using an $L_{1}$ loss, a set of stroke points that accurately reconstructs the original image might still incur a very large loss if the intervals between stroke points do not align well with the GT. Moreover, if the stroke has a small loop, the network might learn to exploit the loss by tending to place stroke points in the middle of the loop to minimize distance to all possible points in the loop if it cannot infer the direction of the stroke. This is likely a less desirable solution than one that predicts points that faithfully reconstruct the loop but in the reverse order of the GT.

Our goal is then to find a better mapping from the predicted points and GTs. Since many different sequences of points can define the same function, we do not constrain our investigation to a bijective mapping, matching each point in our prediction sequence to

precisely one target. Rather, we consider many to many mappings that favor ensuring every predicted point is near a GT point, every GT point is near a predicted point, and the order of the predicted points mirrors that of the GT points. 
Formally, for all $t \in T$, we wish to minimize the distance between $t$ and the nearest $p \in P, p^{*}$. The constraint,

$$
\min _{p \in P}\left\|t_{i}-p\right\|
$$

ensures that our prediction spans the entire GT function $r$.

Similarly, we wish to minimize the distance between any $p \in P$ and the nearest point $t^{*} \in T$, i.e.

$$
\min _{t \in T}\left\|p_{i}-t\right\|
$$

This ensures that each $p$ is proximate to the original function, or that each predicted point lies on the original stroke.

Finally, we wish to find a mapping that preserves the order each point appears within the stroke. Specifically, for $i$ indexing the sequence of points in some stroke and $s$ indexing some mapping, our mapping should require monotonicity

$$
i_{s-1} \leq i_{s}
$$

and continuity

$$
i_{s}-i_{s-1} \leq 1
$$

A fast, dynamic programming algorithm that satisfies these constraints is dynamic time warping (DTW) [22]. DTW is robust to translations and dilations along a dimension, traditionally through the time dimension. Once the sequences are aligned, a loss function can be defined by some distance metric between the aligned points. DTW is typically computed by first computing a cumulative cost matrix, where each cell is the cumulative, minimum cost needed to reach that cell. The last cell then provides the cost of the optimal alignment, while the optimal alignment can be solved with backward induction. The marginal cost matrix is defined as

$$
\Delta(P, T):=\left[\delta\left(P_{i}, T_{j}\right)\right]_{i j} \in \mathbb{R}^{n \times m}
$$


where $i$ and $j$ are indices for the set of predicted points $P$ of size $n$ and target points $T$ of size $m$. This version of the DTW algorithm has $O(n m)$ time complexity. Often, a window is specified, such as the Sakoe-Chiba Band, a uniform warping window that specifies an upper bound $k$ such that $k \geq|i-j|$. which reduces the complexity to $O(n k)$, where $n \geq m$ and $n-m<k$.

For our experiments, we prefer the $L_{1}$ loss, since $L_{2}$ penalizes outliers more and has a tendency to produce more conservative predictions that fail to cover the entire corresponding GT strokes. An example of this might be the following: if the model is uncertain in which direction the cross of a "t" was drawn, it may predict only stroke points in the center of the cross. However, a solution that predicts stroke points that span the entire cross but the wrong direction might be a preferable for many tasks.

\subsection{Adaptive Ground Truth}

In many instances, it is impossible to infer from an image the direction a stroke was drawn, or the order in which the strokes were drawn. As previously alluded to, a writer may cross a "t" with a left-to-right or right-to-left stroke. Similarly, a writer may cross a "t" or dot an "i" immediately or upon the completion of a word or sentence.

This presents a challenge for our stated approach, since the loss function provides better feedback if the system correctly predicts the order and direction of each stroke. However, for many applications, it is more important for the system to predict strokes that reproduce the image of the stroke in high fidelity, while being more invariant to the direction and order of the original strokes.

A potential weakness of employing DTW as our mapping algorithm is that it enforces continuity and monotonicity on the entire sequence of strokes. But while monotonicity and continuity should be enforced within each stroke, we generally wish to relax this requirement for a set of strokes to ensure that pathological strokes do not inhibit the ability of the system 
to make predictions that faithfully reconstruct the image, even if so doing increases the loss we have proposed.

While the order of GT strokes can be adjusted a priori, it is unclear which, if any, orderings would be completely learnable by the system. For instance, a rule might be imposed that dictates the next stroke in a sequence is always that which begins farthest to the left. However, for this and similar rules, there will be cases where different strokes satisfy the criterion comparably well and the system may be unable to consistently recognize the proposed rule. In this case, choosing such a rule would effectively be trading one set of pathological strokes for another.

Moreover, a complete reordering of all strokes may not be as desirable as the original stroke order, or a reasonably similar one, as changing the GT order discards information that the system might have been able to learn and leverage for downstream tasks.

Since we wish to largely preserve sequences of strokes while simultaneously minimizing the number of pathological model updates induced by reversed or out-of-order strokes, we consider a method for permuting stroke order and direction during training. Since training our model requires millions of updates, it is not feasible to perform exhaustive searches over all likely stroke orderings and directions for each update. Moreover, this permutation needs to be recomputed each time the model trains on that instance, as the model could favor a different ordering each epoch.

However, since each instance will be assessed by the model many times, we can adopt an iterative stroke reordering approach. Specifically, for each update, we perform at most one alteration to the GT strokes sequence, either swapping the order of adjacent strokes or inverting the sequence of points of a single stroke. We can identify candidate strokes by those with the greatest total or average DTW loss:

$$
\Delta(P, T):=\left[\delta\left(P_{i}, T_{j}\right)\right]_{i j} \in \mathbb{R}^{n \times m} .
$$


To select a candidate to swap, we perform a softmax on this loss for each stroke to compute the sample probability of that stroke being altered:

$$
\operatorname{Softmax}\left(x_{i}\right)=\frac{\exp \left(x_{i}\right)}{\sum_{j} \exp \left(x_{j}\right)} .
$$

After sampling a stroke, we compute what the loss would have been had the GT stroke been altered by one of three transformations (if applicable): reversing the direction of the stroke, swapping that stroke with the next stroke, or swapping that stroke with the previous stroke. After performing this transformation, we recompute a new alignment and cost for this stroke. If any of the modified GT strokes yield a lower loss, the loss relative to this modified GT stroke is used to update the model, while the change is stored for the next time that exemplar is used for training. As the number of iterations increases and each training instance is trained on multiple times, the model converges to a solution that better reconstructs the original images.

To improve efficiency, we do not need to recompute every possible cost, as the cost matrix prior to the affected stroke has not changed. Additionally, in the same spirit of restricting the possible alignments to a window as described previously, we similarly adopt a window of size $\omega$ which is added to the last index of the alteration. Rather than recomputing the alignment to the end of the sequence, we now compute the alignment only to the end of the stroke plus $\omega$. That is, if the alteration yields a better total cost after the alteration, we consider the alteration to have helped and apply the change.

Because we desire to preserve the actual stroke ordering and directions as much as possible, we employ the adaptive GT method to fine-tune the network after it has largely converged. 


\subsection{Encoder-Decoder Network}

Each offline image is passed through a deep neural network with an encoder-decoder architecture. The encoder encodes salient features of the image in a latent space from which a decoder extracts a probable set of points that correspond to the strokes originally used to create the handwritten characters in the image.

A potential weakness of prior deep learning approaches to this problem, including those described in [13], [25], and [23], is the use of a fixed-length encoder, which inhibits the ability of a model to encode more information as the width of the input increases (to the extent that variable-width inputs can be accommodated in the first place). Consequently, we employ a variable-width encoder based on the Convolution+Recurrent Neural Network (CRNN) architecture, depicted in Figure 3.1. We start with an 11-layer CNN that expects input images to be to 60 pixels tall, 1 channel, and any possible width, to handle handwriting segments of varying length. The output is a matrix of variable width, re-scaled proportionally to the length of the width of the original image, and has a height of 1024, which can be adapted to fit the complexity of the problem. The specific architecture we use is depicted in Figure 3.2. We use $3 \times 3$ kernels for convolution, and both $2 \times 2$ and $2 \times 1$ windows for MaxPool operations.

Each column vector of the matrix is then sequentially fed into a 2-layer, bidirectional, LSTM network that processes feature map data from the CNN, both in a left-to-right and right-to-left order relative to the original image. These outputs from traversing the sequence of data in both directions are then concatenated to each other. The result is a sequence of the same length as the input sequence. The output is passed into a $1 \mathrm{D}$ convolutional layer, which outputs a sequence $S=y_{1}, y_{2}, \ldots y_{M}$. For each step, we predict a relative coordinate $(x, y)$ from the last position, whether the point is the start of a new stroke (SOS token), and whether the point represents the end of a sequence (EOS token).

If the model is trained to predict relative coordinates with no other constraints, the

resulting strokes tend to be reasonably accurate in isolation. However, collectively, these 


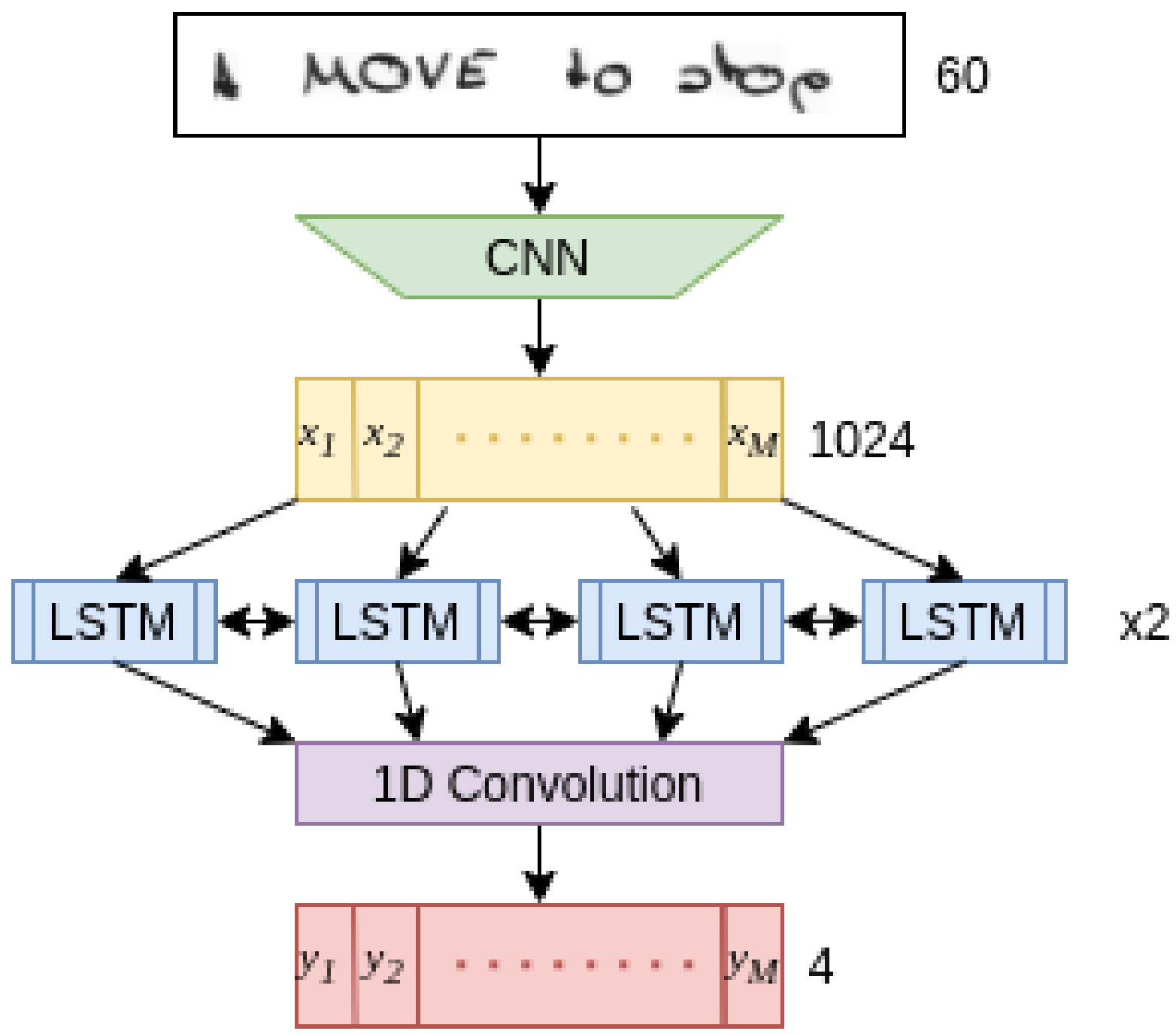

Figure 3.1: The network architecture for TRACE. The input image for the CNN is 60 pixels high with an arbitrary width. The resulting feature maps are approximately the same width as the input and have a height of 1024. This is passed into a 2-layer, bi-directional LSTM, followed by a $1 \mathrm{D}$ convolution. The result is a sequence of stroke point predictions. 


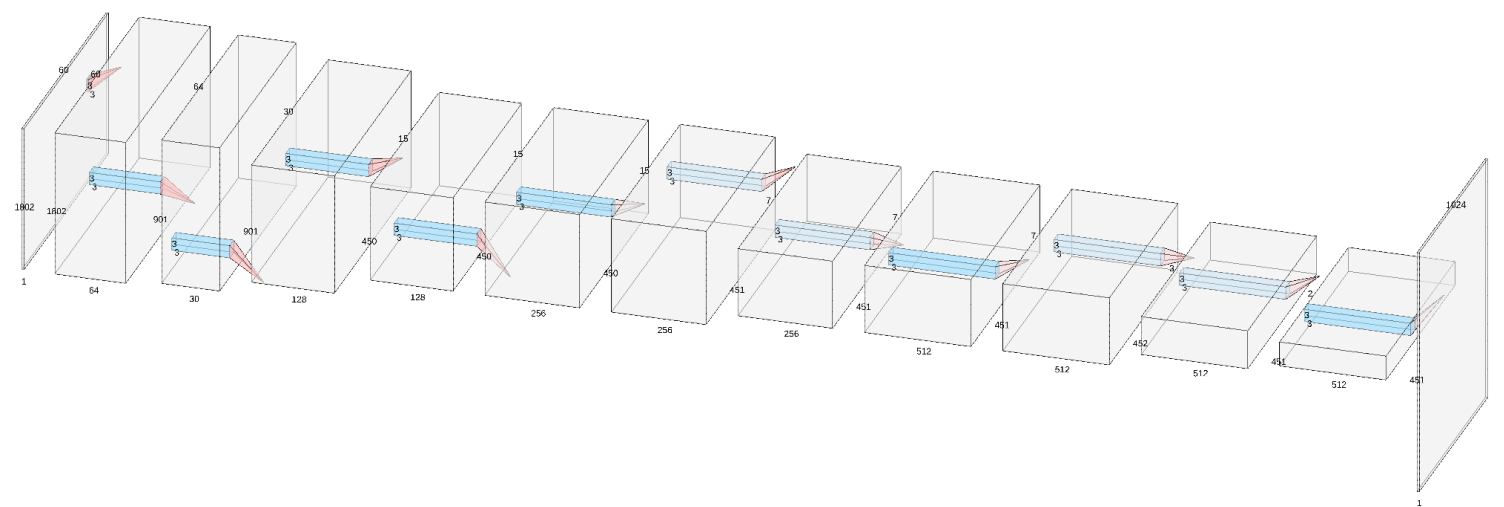

Figure 3.2: Our CNN architecture. We use primarily $3 \times 3$ kernels to perform the series of layer operations: Conv, MaxPool, Conv, MaxPool, Conv, Conv, MaxPool, Conv, Conv, MaxPool, Conv. The output is a matrix of variable width and a height of 1024 .

strokes often do not align well with the input image. This is due, in part, because penup movements are infrequent, and thus more difficult to learn, but simultaneously have a disproportionately large role ensuring the prediction is aligned to the original image. To achieve a kind of global consistency, we compute the cumulative sum of these relative coordinate predictions. We then employ an $L_{1}$ loss to compute the difference between these summed predictions and the GT absolute coordinates.

For predicting SOS tokens, we first compute the DTW alignment between the predicted stroke and GT. Once the alignment is computed, often a particular GT SOS point will match to multiple predicted points. In this case, only the first predicted point will be considered a SOS point when passed to the loss function. We employ a cross-entropy loss with class weights due to the class imbalance between SOS and non-SOS points.

We similarly employ a cross-entropy loss when predicting the EOS. To mitigate the class imbalance issue for EOS tokens, we duplicate the EOS GT stroke point approximately 20 times and append it to the end of the GT stroke sequence. The model is thus trained to predict an EOS token for all successive points after the first EOS stroke point (in contrast to the SOS process described above). This approach allows for the model to learn a smoother 
transition from non-EOS points to EOS points, while also mitigating the class imbalance issue.

For training, we used the ADAM optimizer [12] with a batch size of 32, a learning rate of 0.0001 , and a learning rate schedule that decreased the learning rate at a rate of .96 every 180,000 training instances. 


\section{Chapter 4}

\section{Implementation}

\subsection{Data}

Since our goal is to reconstruct offline handwritten strokes, ideally we would have a set of offline images with corresponding online GT data. However, since these data are comparatively more difficult to collect, we adopt an approach of approximating offline data by rendering online data as images and degrading them. For our experiments, we train our model on 10,426 lines from the IAM online handwriting database (IAM-On) [14], a corpus of trajectory data collected from 221 different writers. To validate the model, we test it on lines from both IAM-On as well as the IAM offline handwriting database (IAM-Off) [15], which is composed of 13,353 lines by 500 different writers.

Each IAM-On line contains a series of positional coordinates $(x, y)$ as well as a time coordinate $t$. Since our model outputs predictions that are proportional to the width of the image, we resample the GT so that the number of stroke points is proportional to the width of the image. Moreover, because we are more concerned with recovering the shape of the strokes than the velocity, the points are resampled with respect to the cumulative stroke distance, so that points within a particular stroke are equidistant. While the model can be used to predict velocity as well by keeping the GT strokes parameterized by time, we prefer to use distance, as generally fewer points are needed to faithfully reconstitute the original strokes.

Each set of strokes is then rendered as an image to be processed by the CNN. To better mimic offline data, a series of augmentations and degradations are applied to each image. 
These include varying stroke width and contrast, as well as applying random grid warping [24], random Gaussian noise, blurring, and other distortions [3]. We further supplement these data with 200,000 synthetic samples generated from a system based on the one described in [8] trained on the IAM-On training data. 


\section{Chapter 5}

\section{Experiments}

We evaluate the success of our model using quantitative metrics for how well it recovers strokes from online data and offline data, as well as evaluating how successful it is in aiding downstream tasks, such as handwriting synthesis using offline data. We establish the first baseline performance for stroke recovery for lines of text on both the IAM-On and IAM-Off data.

\subsection{Online evaluation}

We first consider how successfully the model recovers stroke trajectory data from online data. Since the GT strokes are known, we use the DTW distance score between the GT stroke points and our predicted points (i.e., the cumulative sum of the relative points, as in the loss function). Table 5.1 reports $L_{1}$ and $L_{2}$ average DTW scores, both for the actual GT (where points are sampled as a function time), as well as a resampled version where points are sampled as a function of cumulative stroke distance, as TRACE was trained to predict equidistant stroke points and ignore velocity. Additionally, because TRACE predicts more points than were in the original GT and predicting more points tends to decrease average DTW distances, we resample the predictions to have the same number of points as the original GTs. The DTW distances are scaled so that the distance from the lowest stroke point to the highest stroke point has a unit distance of 1.

Another metric we report is the average distance to the nearest neighbor (NN distance). In this case, we measure the distance between each predicted point and the nearest GT 
Figure 5.1: Random sample of IAM-offline stroke reconstructions

(1) norther. And what has it made of the Congo?

(2) breakfast. It noes a heantifue day, as firsis-of Tue

(3) in Bavaria to witness the Passion Slay. The place and its people were (4) and the temporary assumption of the government by

(5) on express? took him off yesterday!

(6) against the transom. Start at the bow and

(7) be accepted by both East and West.

(8) from outlying districts to stations in

(9) to plants the rides. Start again at the bows,

Table 5.1: Average DTW distance

\begin{tabular}{|c|c|c|}
\hline \multirow{2}{*}{ Distance metric } & \multicolumn{2}{|c|}{ Average DTW loss } \\
\cline { 2 - 3 } & (equidistant GT) & (original GT) \\
\hline \hline$L_{1}$ & 0.03060 & 0.03452 \\
\hline$L_{2}$ & 0.02423 & 0.02745 \\
\hline
\end{tabular}

Table 5.2: Online NN distance

\begin{tabular}{|c|c|c|}
\hline \multirow{2}{*}{ Type } & \multicolumn{2}{|c|}{ Average NN distance $\left(L_{2}\right)$} \\
\cline { 2 - 3 } & (equidistant GT) & (original GT) \\
\hline \hline GT to nearest prediction & 0.01662 & 0.01751 \\
\hline Prediction to nearest GT & 0.01405 & 0.01615 \\
\hline
\end{tabular}

19 
Table 5.3: Offline NN distance

\begin{tabular}{|c|c|}
\hline Type & Average NN distance $\left(L_{2}\right)$ \\
\hline \hline Prediction to nearest GT & $9.311 \times 10^{-4}$ \\
\hline
\end{tabular}

point and vice versa. Measuring each predicted point to the nearest GT truth is a measure somewhat akin to precision, and measures the extent to which the predicted points lie somewhere on the GT. Conversely, measuring the distance between each GT point to the nearest prediction resembles recall, and measures how well the predictions cover the entire space of GT points. Table 5.2 suggests that TRACE is slightly better at ensuring predicted stroke points are near GT stroke points than it is at ensuring every GT stroke point is near a predicted point, which is supported by the observation that TRACE, e.g., does not cross every "t". As with DTW loss, using equidistant GT points decreases error.

Note that shifting predicted points nearer to the GT point could be done with both online and offline data as a post processing step to reduce these errors. We present results without any such post-processing to provide a baseline for the differentiable method we have proposed.

\subsection{Offline evaluation}

Because IAM-Off does not have ground truth strokes, we consider only the average NN distance from each predicted point to the nearest GT pixel, as opposed to the nearest stroke point. In this case, we define a GT pixel on the image as one that has an intensity of less than 127.5 on a scale from 0 to 255 , which creates many more GT points of comparison than in the online experiment. This result is reported in Table 5.3.

Qualitative results of the process can be observed in Figure 5.1, which shows a random sample of GT offline images with the recovered strokes overlaid in red. TRACE tends to do very well in predicting neat, well-spaced handwriting, and generally better when the handwriting stands in high contrast to the background. TRACE struggles somewhat with 


\section{th now took an ominons squificance: Nigel had}

Figure 5.2: TRACE achieves robust performance despite the presence of an anomalous marking.

predicting punctuation, as well as isolated strokes, or strokes that are often not drawn consecutively with respect to the rest of the character (as in the dot in an "i" or the cross in a "t"). Also, it sometimes fails to accurately predict stroke extremities, or strokes that approach too near the top of the line (as in the "P" in "Passion" on line 3).

Figure 5.2 shows it is somewhat robust to anomalies as it successfully resumes after an aberrant, scribbled out marking.

\subsection{Synthesis evaluation}

While recovering handwritten stroke trajectory may be of interest for its own sake, often it is considered an intermediate step for solving other handwriting tasks, including handwriting recognition, handwriting synthesis, and writer verification. While there are many possible ways to incorporate recovered strokes into these systems, we demonstrate one way it can be done for handwriting synthesis.

One of the first and perhaps most famous handwriting synthesis models is described in [8]. In this model, an LSTM is used to parameterize a Mixture Density Network (MDN), which can then be iteratively sampled to predict each successive stroke point. A feature of using an LSTM is that it has a vector that defines the "state" of the LSTM. In general, this state allows the network to learn both short and long-term dependencies, and in the case of [8], enables the model to parameterize the MDN in a manner that mimics the style of the data used to "prime" the model. That is, the model has some ability to synthesize text in any style as defined by the state vector of the LSTM. Naturally, the ability for the LSTM to define a state that embodies a particular style improves with the number of samples of that style used during training. 
Part of the success of this particular model depends on the input data being structured as a sequence of points, rather than as an image, as in the case of offline handwriting. While this model was successful in synthesizing new text from online data, there was no straightforward way to apply it to offline data. In our case, we use recovered strokes from offline data to variously train or prime the model to synthesize offline handwriting styles.

Using offline handwriting in this system is useful not only for cases when the target handwriting style has not been captured online, but also because online and offline handwriting styles are not the same. Factors that contribute to these differences include the friction between the writing surface and implement, the responsiveness and sensitivity of the digital screen, and any changes to the way a person holds his or her hand when writing on a digital surface (e.g., so as to not touch the screen with the side of his or her hand). Moreover, strokes recovered from offline text can be used as a way to augment the training data of these kinds of systems, which improves generalization.

Figure 5.3 demonstrates the ability of the synthesis system to mimic the style of an offline sample. Note that all synthetic texts are rendered with the same stroke width and consequently mimic only the rough shape of the original input and not, e.g., line quality. For synthesizing experiments, we synthesize English pangrams, sentences that include every letter of the alphabet at least once. Our test sentences include

- Sphinx of black quartz, judge my vow.

- The five boxing wizards jump quickly.

- How vexingly quick daft zebras jump.

As Figure 5.3 shows, more common letters and n-grams generally appear to produce better results. 
(a) exercise any degree of control over the action Sphinx of black quartz, judge my vow

(b) At the same time that unity cannot be How vexingly quick daft zebras jump

(c) It went, perhaps, some distance beyond Spleens of Each quarts, judge my vow (d) Scot - and so were four of his predecessors this century. The five boxing wizards jump quickly

Figure 5.3: Synthetic online handwriting samples inspired from offline styles. Each pair of lines above constitutes (1) an offline image used to prime the synthetic text model and (2) an image of synthetic text generated by the model in the style of the offline image.

(a) and the polished brass fousteen-pounder shell -case

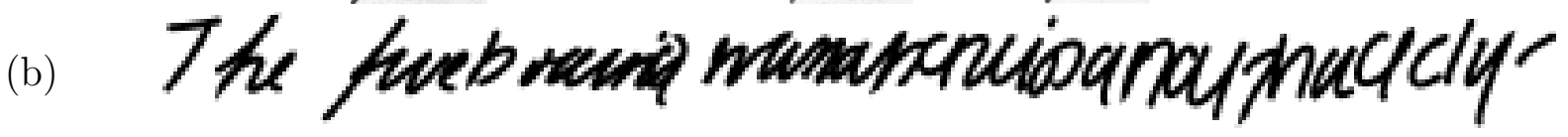

(c) The fir laving word prop queenly

(d) The fur boxing uspards jump quickly

Figure 5.4: Comparison of synthesized text based on different training data. (a) is a sample of offline handwriting data used to prime the handwriting synthesis model. (b), (c), and (d) are results from models trained on only online data, only offline data, and both online and offline data, respectively. The synthetic text is the pangram "The five boxing wizards jump quickly."

23 


\subsection{Synthetic Evaluation}

Figure 5.4 compares text generated by a system trained only with online data, another with only converted offline data, and another one trained on both, all being primed with a converted offline sample. While all three achieve various success in this task generally, the system is more prone to produce worse or degenerate samples when primed with a style it has not been trained on, as noted in [8].

Note that the inability of the synthesis system trained only on native online data to synthesize text when seeded with converted offline data may be due, in part, because of imperfections that arise from the conversion process, though it may also be related to inherent distinctions between online and offline handwriting styles. When the system is trained on offline data, and particularly when it is trained on other samples of a particular author, the system produces better synthetic samples. Generally, the system trained on both the IAM-On data and our reconstructed IAM-Off data produces the best synthetic samples. Thus, not only does our system enable seeding the synthesis model with an offline sample in the first place to mimic an offline handwriting style, it also augments the set of training data available to the synthesis model, enabling it to produce better synthetic handwriting samples.

\subsection{Adaptive GT Ablation Study}

To demonstrate how the adaptive GT can improve the system, we first train the network for 50 epochs with the original GTs. The intent is to have the system first learn the GT stroke orders and directions and afterward fine-tune with adaptive GTs to improve the system's ability to handle more anomalous strokes.

Once the network has been pretrained, we employ the adaptive GT algorithm described in section III. Figure 5.5 shows how the number of swaps and changes is small to begin with, as a change to the GT is helpful for fewer than 1 in 10 instances. After 40 additional epochs, the number of changes has started to converge with $35 \%$ fewer changes per instance than 


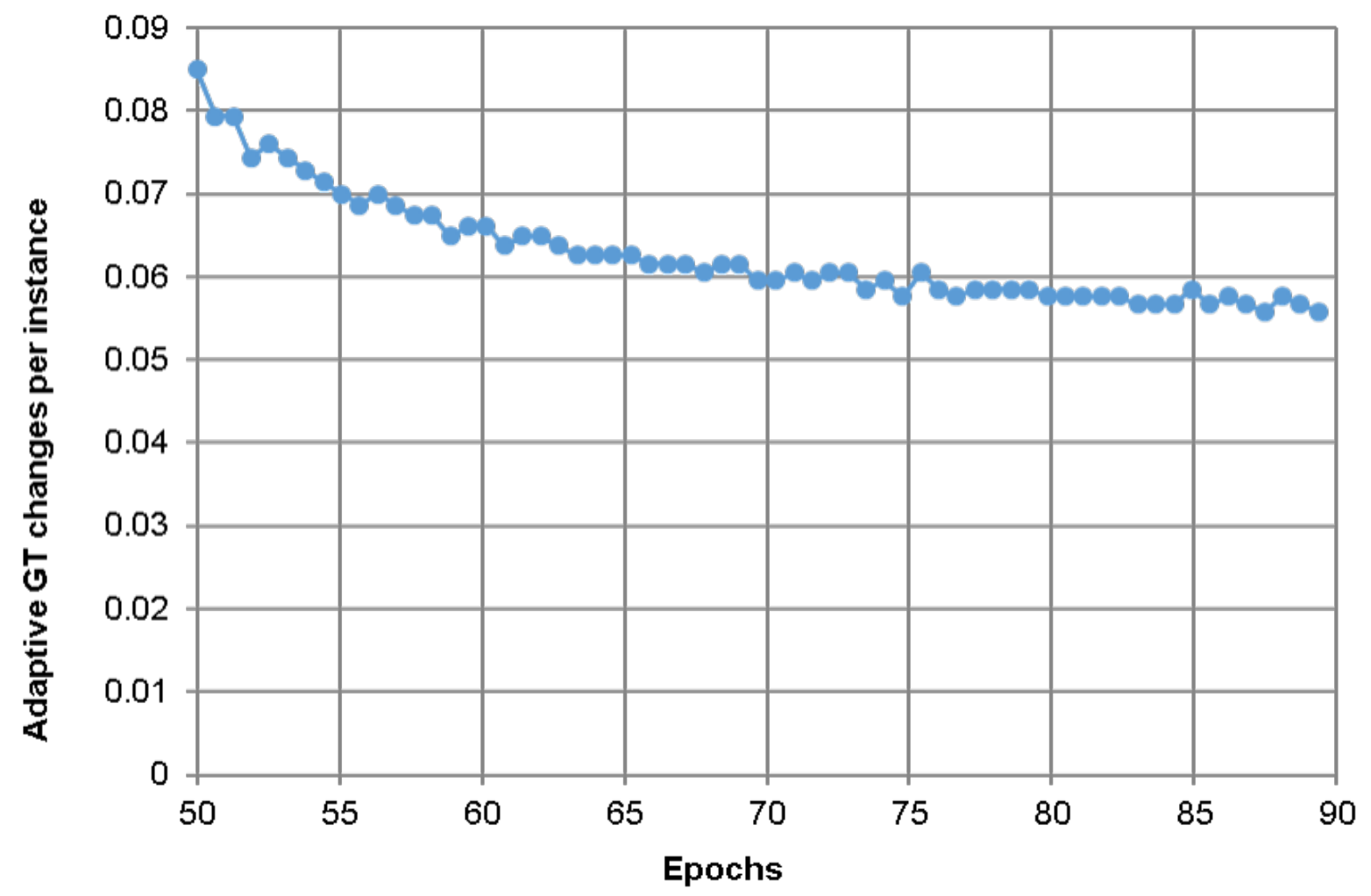

Figure 5.5: The number of GT adaptive improvements per training instance converges. 


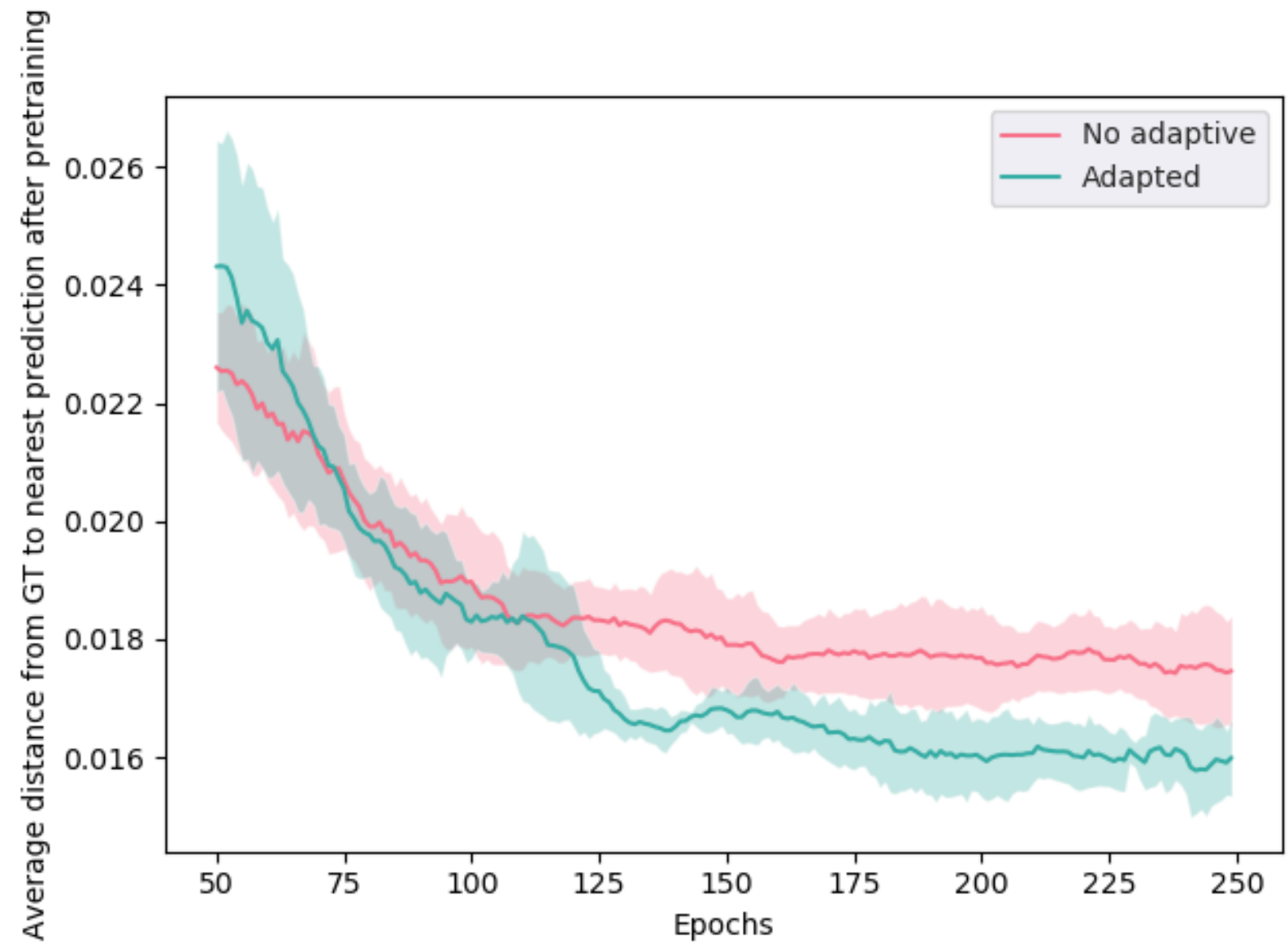

Figure 5.6: Adapting GTs on the training set improves NN loss on test set.

initially. Figure 5.6 shows how the average NN loss on 5 runs converges to a lower loss than without the use of adaptive GTs on the test set. 


\section{Chapter 6}

\section{Conclusion}

In this work, we have proposed TRACE, a novel method to recover stroke trajectories from offline data, and, in effect, rendering it in an online format. TRACE works well on wide images composed of many characters and strokes and is completely differentiable. We have demonstrated that it can be used to enable online handwriting synthesis to work with offline data.

There are several possible directions for future work. On the applications side, TRACE can be used as a loss function for synthesizing an offline handwriting image directly, as opposed to synthesizing strokes only, and could supplement approaches presented in [5] and [7]. Another possibility would be testing the extent recovered strokes can be used to augment training data for online recognition systems.

To improve the stroke recovery method, one direction might be to use a generative model, such as training a mixture density network as in [8, 9], or an invertible neural network $[1,6]$. This would afford the model greater ability to model uncertainty and provide multimodal solutions. Another possibility would be experimenting with preprocessing techniques, such as reparameterizing input strokes as Bézier curves, as in [4]. 


\section{References}

[1] Lynton Ardizzone, Jakob Kruse, Carsten Rother, and Ullrich Köthe. Analyzing inverse problems with invertible neural networks. In International Conference on Learning Representations, 2019. URL https://openreview .net/forum?id=rJed6j0cKX.

[2] Ayan Kumar Bhunia, Subham Mukherjee, Aneeshan Sain, Ankan Kumar Bhunia, Partha Pratim Roy, and Umapada Pal. Indic handwritten script identification using offline-online multi-modal deep network. Information Fusion, 2019.

[3] T. M. Breuel. Tutorial on ocr and layout analysis. Technical report.

[4] Victor Carbune, Pedro Gonnet, Thomas Deselaers, Henry A Rowley, Alexander Daryin, Marcos Calvo, Li-Lun Wang, Daniel Keysers, Sandro Feuz, and Philippe Gervais. Fast multi-language lstm-based online handwriting recognition. International Journal on Document Analysis and Recognition (IJDAR), pages 1-14, 2020.

[5] Brian Davis, Chris Tensmeyer, Brian Price, Curtis Wigington, Bryan Morse, and Rajiv Jain. Text and style conditioned gan for generation of offline handwriting lines. 31st British Machine Vision Conference, BMVC, 2020.

[6] Laurent Dinh, Jascha Sohl-Dickstein, and Samy Bengio. Density estimation using Real NVP. 5th International Conference on Learning Representations, ICLR 2017 Conference Track Proceedings, may 2016. URL http://arxiv.org/abs/1605.08803.

[7] Sharon Fogel, Hadar Averbuch-Elor, Sarel Cohen, Shai Mazor, and Roee Litman. Scrabblegan: Semi-supervised varying length handwritten text generation. In Proceedings of the IEEE/CVF Conference on Computer Vision and Pattern Recognition, pages 43244333, 2020.

[8] Alex Graves. Generating sequences with recurrent neural networks. arXiv preprint arXiv:1308.0850, 2013.

[9] Alex Graves. Stochastic Backpropagation through Mixture Density Distributions. jul 2016. URL http://arxiv.org/abs/1607.05690. 
[10] Sepp Hochreiter and Jürgen Schmidhuber. Long short-term memory. Neural computation, 9(8):1735-1780, 1997.

[11] D. Keysers, T. Deselaers, H. A. Rowley, L. Wang, and V. Carbune. Multi-language online handwriting recognition. IEEE Transactions on Pattern Analysis and Machine Intelligence, 39(6):1180-1194, 2017.

[12] Diederik P. Kingma and Jimmy Lei Ba. Adam: A method for stochastic optimization. In 3rd International Conference on Learning Representations, ICLR 2015 - Conference Track Proceedings. International Conference on Learning Representations, ICLR, dec 2015. URL https://arxiv.org/abs/1412.6980v9.

[13] Ayan Kumarbhunia, Abir Bhowmick, Ankan Kumar Bhunia, Aishik Konwer, Prithaj Banerjee, Partha Pratim Roy, and Umapada Pal. Handwriting Trajectory Recovery using End-to-End Deep Encoder-Decoder Network. In Proceedings - International Conference on Pattern Recognition, volume 2018-August, pages 3639-3644. Institute of Electrical and Electronics Engineers Inc., 11 2018. ISBN 9781538637883. doi: 10.1109/ICPR.2018.8546093.

[14] Marcus Liwicki and Horst Bunke. IAM-OnDB - An on-line English sentence database acquired from handwritten text on a whiteboard. In Proceedings of the International Conference on Document Analysis and Recognition, ICDAR, volume 2005, pages 956-961, 2005. ISBN 0769524206. doi: 10.1109/ICDAR.2005.132.

[15] U-V Marti and Horst Bunke. The iam-database: an english sentence database for offline handwriting recognition. International Journal on Document Analysis and Recognition, $5(1): 39-46,2002$.

[16] E-M Nel, Johan A Du Preez, and Ben M Herbst. Estimating the pen trajectories of static signatures using hidden markov models. IEEE transactions on pattern analysis and machine intelligence, 27(11):1733-1746, 2005.

[17] Vu Nguyen and Michael Blumenstein. Techniques for static handwriting trajectory recovery. In Proceedings of the 8th IAPR International Workshop on Document Analysis Systems - DAS '10, pages 463-470, New York, New York, USA, 2010. ACM Press. ISBN 9781605587738. doi: 10.1145/1815330.1815390. URL http://portal .acm.org/ citation. cfm?doid=1815330 1815390 .

[18] Réjean Plamondon and Claudio M. Privitera. The segmentation of cursive handwriting: An approach based on off-line recovery of the motor-temporal information. IEEE 
Transactions on Image Processing, 8(1):80-91, 1999. ISSN 10577149. doi: 10.1109/83. 736691.

[19] Réjean Plamondon and Sargur N. Srihari. On-line and off-line handwriting recognition: A comprehensive survey. IEEE Trans. Pattern Anal. Mach. Intell., 22(1):63-84, January 2000. ISSN 0162-8828. doi: 10.1109/34.824821. URL https://doi.org/10.1109/34. 824821.

[20] Rejean Plamondon, S.N. Srihari, and Sargur N. Srihari. On-Line and Off-Line Handwriting Recognition: A Comprehensive Survey. IEEE Transactions on Pattern Analysis and Machine Intelligence, 22(1):63-84, 2000. ISSN 01628828. doi: 10.1109/34.824821. URL http://ieeexplore.ieee.org/document/824821/.

[21] G. Ren and V. Ganapathy. Recognition of online handwriting with variability on smart devices. In ICASSP 2019 - 2019 IEEE International Conference on Acoustics, Speech and Signal Processing (ICASSP), pages 7605-7609, 2019.

[22] Hiroaki Sakoe and Seibi Chiba. Dynamic programming algorithm optimization for spoken word recognition. IEEE transactions on acoustics, speech, and signal processing, 26(1):43-49, 1978.

[23] Taichi Sumi, Brian Kenji Iwana, Hideaki Hayashi, and Seiichi Uchida. Modality conversion of handwritten patterns by cross variational autoencoders. In 2019 International Conference on Document Analysis and Recognition (ICDAR), pages 407-412. IEEE, 2019.

[24] Curtis Wigington, Seth Stewart, Brian Davis, Bill Barrett, Brian Price, and Scott Cohen. Data Augmentation for Recognition of Handwritten Words and Lines Using a CNN-LSTM Network. In Proceedings of the International Conference on Document Analysis and Recognition, ICDAR, volume 1, pages 639-645. IEEE Computer Society, 1 2017. ISBN 9781538635865. doi: 10.1109/ICDAR.2017.110.

[25] Bocheng Zhao, Minghao Yang, and Jianhua Tao. Pen Tip Motion Prediction for Handwriting Drawing Order Recovery using Deep Neural Network. In Proceedings International Conference on Pattern Recognition, volume 2018-Augus, pages 704-709. Institute of Electrical and Electronics Engineers Inc., 11 2018. ISBN 9781538637883. doi: 10.1109/ICPR.2018.8546086. 